\title{
Growth and Characterization of New Non Linear Optical Bis-Glycine Hydro Bromide (BGHB) Single Crystal
}

\author{
S. Sampthkrishnan ${ }^{\text {* }}$, N. Balamurugan ${ }^{\mathrm{b}}$, R. Kumutha ${ }^{\mathrm{c}}$, Y. Vidyalakshmi ${ }^{\mathrm{d}}$, S. Muthu ${ }^{\mathrm{a}}$ \\ ${ }^{a}$ Department of Physics, Sri Venkateswara College of Engineering, Sriperumbudur, India. \\ ${ }^{\mathrm{b}}$ Department of Physics, PERI Institute of Technology, Chennai-600 048, India. \\ ${ }^{\mathrm{c}}$ Department of Physics, JAYA Engineering College, Chennai, India. \\ ${ }^{\mathrm{d}}$ Departments of Physics, MIT, Anna University, Chennai-48. \\ *Corresponding Author: Sambathk@s,svce.ac.in, n_rishibalaa@yahoo.co.in
}

\begin{abstract}
A new non linear optical material, Bis-Glycine Hydro bromide (BGHB), has been synthesized. Single crystals of BGHB have been grown successfully by slow evaporation method. The solubility of the material was measured in various solvents such as ethanol, acetone and water. It was found to have extremely low solubility in ethanol and acetone. The grown crystals were characterized by recording the powder diffraction and identifying the diffracting planes. Using single crystal diffractometer the morphology of BGHB crystal was identified. Fourier transform infrared (FTIR) spectroscopic studies, optical behavior such as UV-visible-NIR absorption, Thermogravimetic (TG) and differential scanning calorimetric (DSC) analyses have been performed to show that $B G H B$ is thermally stable up to $168.5^{\circ} \mathrm{C}$ and there is no phase transition and decomposition till $168.5^{\circ} \mathrm{C}$. Anisotropy in the hardness behavior has been observed while measuring at different crystal planes by Vicker hardness test.
\end{abstract}

Key words: Solubility, Grown from solution, Crystal structure, FT-IRspectroscopy, Micro hardness,

\section{INTRODUCTION}

In the technological society, development of new devices has been introduced through the growth of single crystals. Crystals for practical and technological applications should have a well developed morphology and contain low density of defects predicted by thermodynamic and 
kinetic parameters which determine the growth mechanism and the growth kinetics and the generation of defects respectively. This method is extremely popular in the production of many technologically important crystals. Much organic and inorganic crystal can be grown using this technique [1]. Most of the organic NLO crystals usually have poor mechanical and thermal properties and are susceptible for damage during processing even though they have large NLO efficiency. Also, it is difficult to grow larger size optical-quality crystals of these materials for device applications. Purely inorganic NLO materials have excellent mechanical and thermal properties, but possess relatively moderate optical nonlinear property because of the lack of extended $\pi$-electron delocalization [2, 3]. L-Cysteine hydrochloride [4], L-Histidine hydrochloride [5], L-Histidine tetrafluroborate [6], L-Histinium bromide [7], L-Histidine hydrofluride dehydrate [8] and L-Glutamic acid hydrocholoride (GHC) [9] are some examples of semiorganic amino acid-related NLO materials reported recently. Presently, the single crystals of a new semiorganic NLO material viz., Bis-Glycine hydrobromide (BGHB) have been grown by slow evaporation method. In the present work, the solubility of BGHB was measured in different solvents. The growth aspects, morphology, the results of the XRPD and FTIR analysis have been discussed. The differential scanning calorimeter (DSC), DifferentialThermalAnalysis (TGA), thermogravimetric (TGA) analysis and SHG efficiency of the powdered materials was measured by using the Kurtz and Perry method [10]. Measurements of microhardness are also reported.

\section{EXPERIMENTAL PROCEDURE}

\subsection{Crystal Growth}

High purity Glycine salt (E-merck) and hydrobromic acid (E-Merck) were taken in the molar ratio 2:1 in deionized water to synthesis BiGlycine Hydrogen Bromide salt. The $\mathrm{pH}$ of the solution was adjusted to be 2 and the growth experiment was maintained at $318 \mathrm{~K}$. The saturated BGHB solution of $\mathrm{pH} 2$ has been prepared using doubly recrystallised salt. The solution was filtered using sintered glass filter $1 \mu$ porosity. The filtered solution was transferred into the petty disc and allowed to evaporate slowly at room temperature. Transparent and flawless crystals size: $(22 \times 6 \times 8) \mathrm{mm}^{3}$ were obtained after 10 days as shown in the Figure (1).

\subsection{Solubility of BGHB}

The solubility of BGHB was measured for different solvents (ethanol, methanol and acetone) by gravimetrical analysis method. Water has been selected as a solvent, because the solubility of BGHB is more in water compared with other solvents. The aqueous solution of recrystallised BGHB was prepared and maintained at constant temperature with continuous stirring to ensure homogeneous temperature and concentration over the entire volume of the solution. On reaching saturation, the content of the solution was analyzed gravimetrically. The same procedure was repeated for various temperatures such as $30^{\circ} \mathrm{C}, 35^{\circ} \mathrm{C}, 40^{\circ} \mathrm{C}, 45^{\circ} \mathrm{C}$ and $50^{\circ} \mathrm{C}$. The plotted 
solubility curve is as shown in the figure (2). From the figure, it is evident that the solubility increases with increase in temperature.

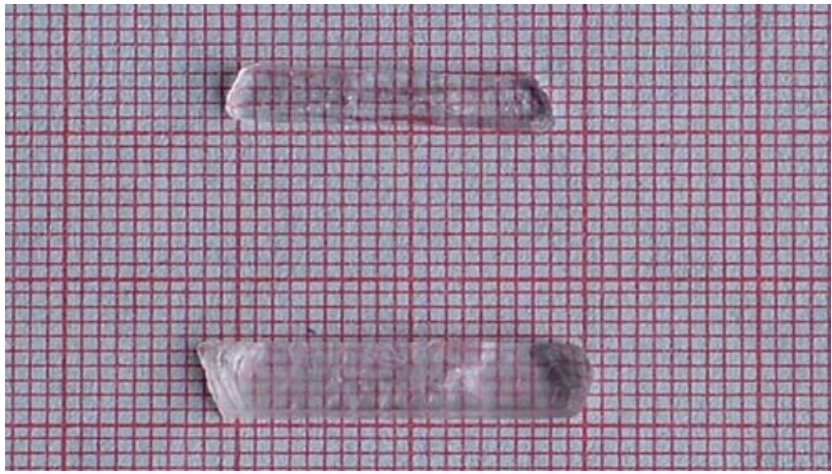

Fig.1: Photograph of BGHB crystal.

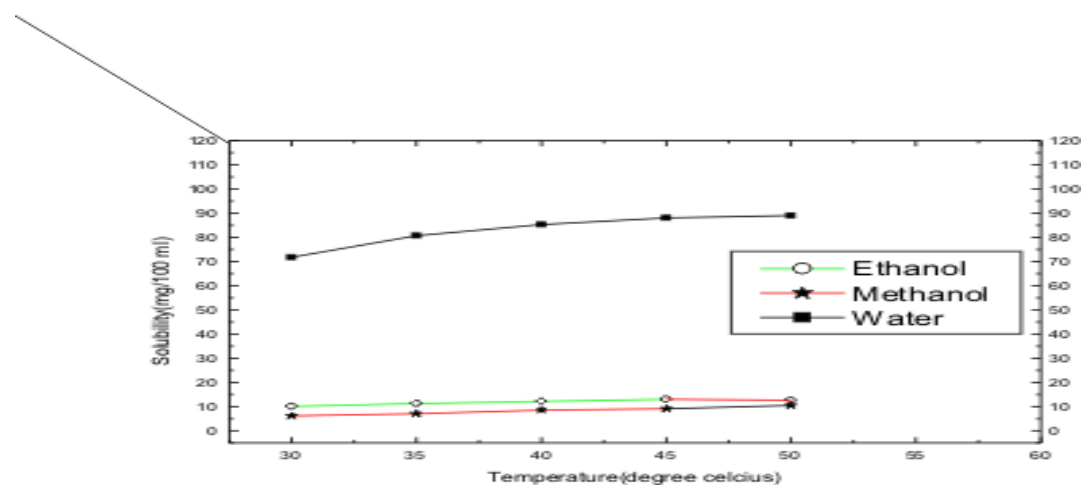

Fig.2. Solubility spectrum of BGHB single crystal

\section{CHARACTERIZATION}

\subsection{Structure Elucidation using Single Crystal X-ray Diffraction}

Single crystal powder X-ray diffraction pattern was recorded using a Siemens D500 diffractometer at room temperature $(293 \mathrm{~K})$ with $\mathrm{CuKa}(X=1.5418 \mathrm{~A})$ radiation for structural analysis of Bi-Glycine Hydrogen Bromide and the lattice parameters were calculated.

\subsection{UV_Vis_Spectroscopic}

To know the optical transparency of the grown crystal, the UV_Vis absorbance spectrum of BHGB was recorded.using a carry 5E UV_Vis_NIR spectrophotometer. In the range between 200 and $2000 \mathrm{~nm}$ 


\subsection{FT-IR Spectroscopic}

The FTIR spectrum of BGHB crystals was recorded in the range near $2000-1800 \mathrm{~cm}^{-1}$ by employing a Brukker IFS66 V FTIR spectrometer by KBr pellet technique to study the presence of glycine in the sample qualitatively.

\subsection{Microhardness Studies}

Microhardness measurements were carried out on BGHB crystal using a Leitz Weitzler hardness tester fitted with a diamond pyramidal indentor. The well polished crystal moulted on the platform of microhardness crystal and loads of different magnitudes (5, 10, 20, 30, 40, and 45) were applied over a fixed interval of time. The indentation time was fixed as $5 \mathrm{~s}$.

\subsection{Thermal Analysis}

Thermal analysis was used to find out the weight loss (TGA) and energy change (DTA) in the sample with respect to the temperature. The thermal analysis was carried out on the powder specimen of BGHB by employing a MettlerToledoStar simultaneous DTA/TGA analyzer at $10^{\mathrm{oc}} / \mathrm{min}$ heating rate in the nitrogen atmosphere by using an alumina crucible.

\section{RESULTS AND DISCUSSION}

\subsection{Crystal Morphology \& Single Crystal X-ray Structure Analysis}

Morphology measured using single crystal XRD reveals that there are well- developed faces (102) and their freidels. The indices of the faces are shown in fig (3). In all grown crystals, the most prominent face is $\left(\begin{array}{lll}0 & 1 & 2\end{array}\right)$. The width of the crystal is in the $\mathrm{C}$ direction. To identify the morphology of the grown crystal, the Single crystal XRD was taken and the data were collected using an ENRAF NONIUS CAD4 automatic X-ray diffractometer.

The physical crystal had the shape of parallelepiped. The line of intersection of the faces (012) and (01-2) is length of the crystal. The line of intersection is $\left(a^{*}+b^{*+} 2 c^{*}\right) \times\left(0 a^{*}+b^{*}-2 c^{*}\right)=-$ $4 \mathrm{v}^{*} \mathrm{a}$. Hence it is inferred that the length of crystal is a -direction which is also the shortest unit cell axis of the crystal. (010) and (001) planes are not morphologically developed.

The XRD pattern and diffraction indices of the crystal are shown in Fig. 4. The XRPD pattern showed that the synthesized material and the as grown crystals are the single phase of BGHB. The orthorhombic unit cell parameters calculated by TREOR program are $a=5.39 \mathrm{~A}^{0}, b=8.18 \mathrm{~A}^{0}$, $\mathrm{c}=18.39 \mathrm{~A}^{0}, \alpha=90.18^{0}, \beta=89.88^{0}, \gamma=89.99^{\circ}$ and $\mathrm{V}=812.4 \mathrm{~A}^{03}$, which are comparable with the results determined by a $\mathrm{R} 3 \mathrm{~m} / \mathrm{E}$ four circle $\mathrm{X}$-ray diffractor . 


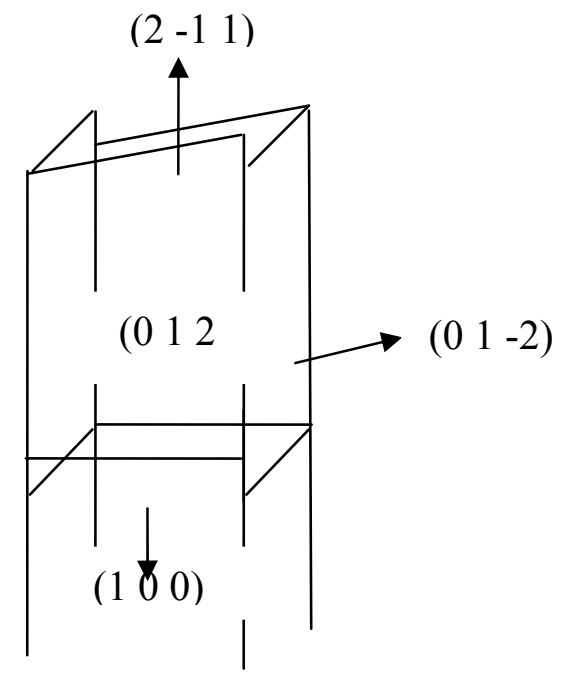

Fig. 3: Morphology of the grown BGHB crystal

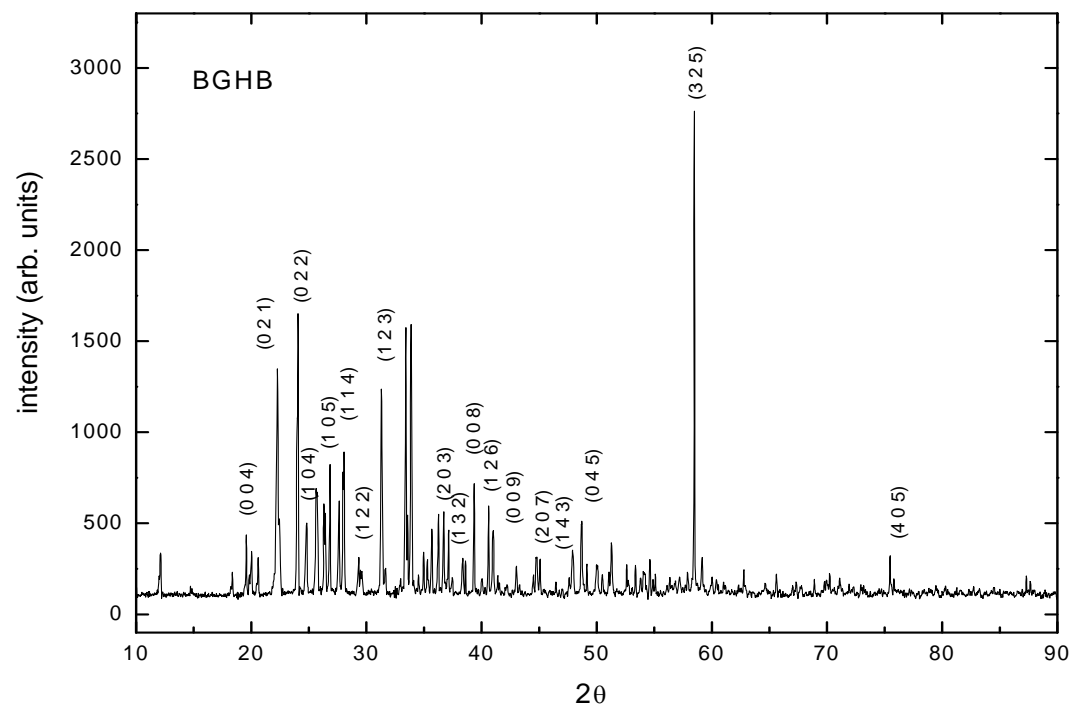

Fig. 4: Powder XRD pattern of BGHB single crystal

\subsection{UV-Vis -NIR Spectrum}

The UV-Vis-NIR transmission spectrum (Fig. 5) of the crystal was reorded in the range: 200 2000nm using carry 5E UV-Vis_NIR spectrophotometer. It is seen that a strong absorption band occurs at $750 \mathrm{~nm}$ and this absorption is due to the $\mathrm{n}->\pi^{*}$ transition. The lower cutoff wavelength occurs at $250 \mathrm{~nm} \& 1600 \mathrm{~nm}$ is due to $\pi->\pi^{*}$ transition [11]. After that, no absorption takes place in the entire visible region. 


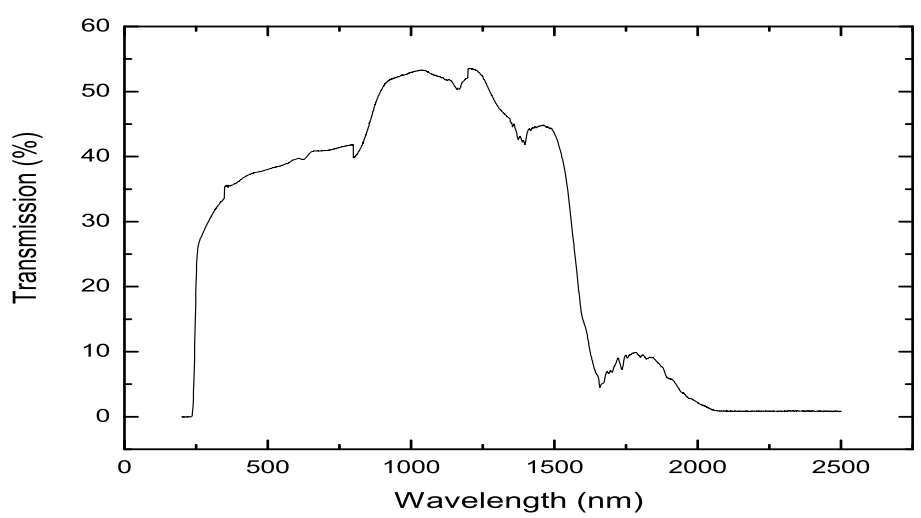

Fig.5: UV-vis-NIR spectrum of BGHB single crystal

\subsection{FT-IR Studies}

To identify the elements and the functional groups present in the grown crystal qualitatively, the FTIR spectra were obtained using Brukker IFS66 V FTIR spectrometer by KBr pellet technique. In the FTIR spectrum shown in Figure (6), there is a broad band between 2000 and $3500 \mathrm{~cm}^{-1}$. The sharp peak at 3429, 3113 and $2897 \mathrm{~cm}^{-1}$ may be assigned to $\mathrm{NH}_{3}{ }^{+}$stretching band. The peaks at $2694 \mathrm{~cm}^{-1}$ and $2601 \mathrm{~cm}^{-1}$ are attributed to the $\mathrm{C}-\mathrm{H}$ stretching mode vibration. The overtone region contains a band near $2000-1800 \mathrm{~cm}^{-1}$ which is assigned to the combination of the asymmetrical $\mathrm{NH}_{3}{ }^{+}$bending and the torsional oscillation of the $\mathrm{NH}_{3}{ }^{+}$groups. The intense peaks at 1743,1715 and $1125 \mathrm{~cm}^{-1}$ indicate the $\mathrm{C}=\mathrm{O}$ stretching of the $\mathrm{COO}^{-}$group. The asymmetric and symmetric stretching mode of $\mathrm{COO}^{-}$groups are reached by peaks at 1616 , 1497, 1446, 1335 and $1254 \mathrm{~cm}^{-1}$. This observation confirms that one glycine is existing in zwitterionic form the peak at $671 \mathrm{~cm}^{-1}$ is due to $\mathrm{N}-\mathrm{H}$ out-of-plane bending vibration. The torsional oscillation of $\mathrm{NH}_{3}{ }^{+}$is revealed by the peak $540 \mathrm{~cm}^{-1}$. The strong carbonyl absorption at $1743 \mathrm{~cm}^{-1}$ characterizes $\alpha$-amino acid hydro bromides.

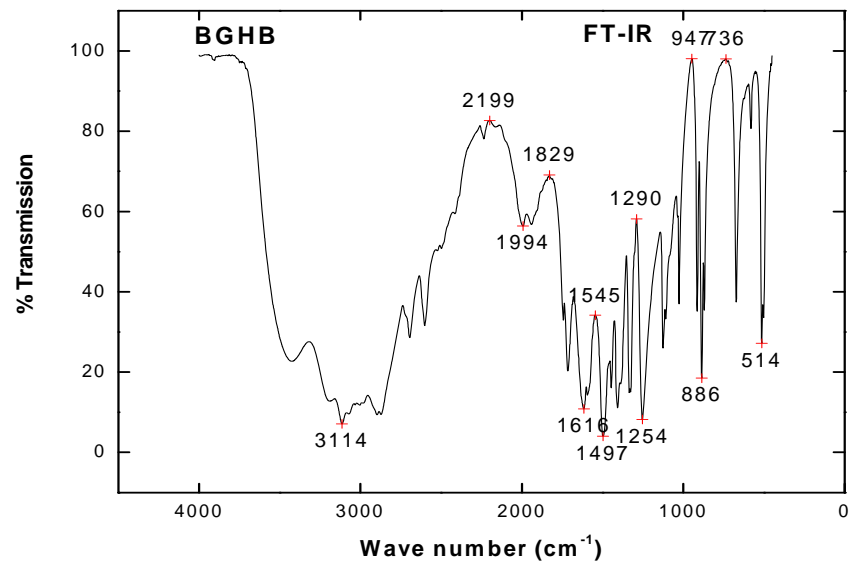

Fig .6.FTIR spectrum of BGHB single crystal 


\subsection{Micro Hardness Studies}

Micro hardness measurements were carried out on BGHB crystals. Smooth surface of BGHB was subjected to the Vickers static indentation test at room temperature (303k) using a Leitz Wetzlar hardness tester fitted with a Vickers diamond pyramidal indenter. Loads of different magnitudes $(5,10,20,30,40$ and 45g) were applied over a fixed interval of time. The indentation time was kept as $5 \mathrm{~s}$ for all the loads. The Vicker micro hardness number was evaluated from the relation

$$
\mathrm{H}_{\mathrm{v}}=(1.6180 \mathrm{p}) / \mathrm{d}^{2} \mathrm{~kg} / \mathrm{mm}^{2} \quad\left(\theta=108^{0}\right)
$$

(Where $\mathrm{p}$ is the applied load in $\mathrm{kg}$ and $\mathrm{d}$ is the diagonal length of the indentation impression in micrometer).

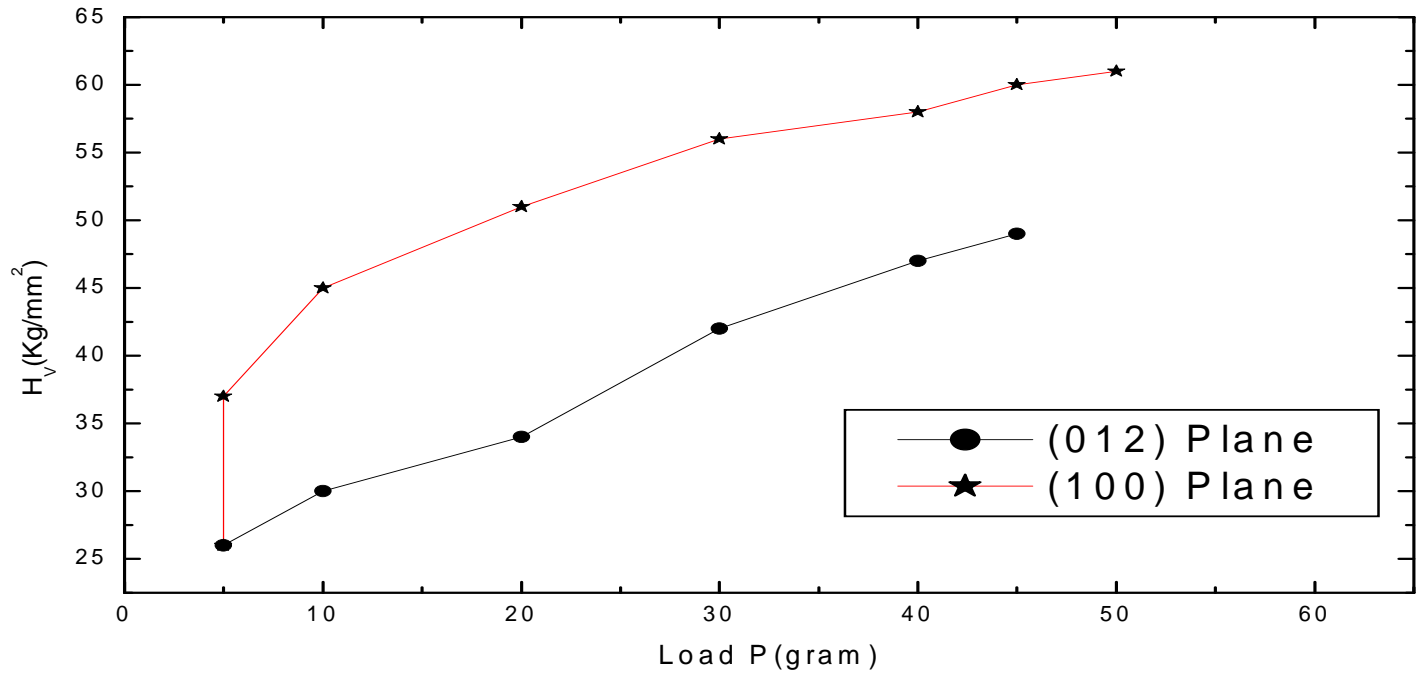

Fig. 7: Micro hardness spectrum of BGHB single crystal

\subsection{Second Harmonic Generation}

The Kurtz powder SHG test confirms the NLO property of the grown BGHB crystals [13]. The relative conversion efficiency was calculated from the output power of BGHB crystals with reference to BGHB and KDP crystals output power for various input powers. Table 1 shows the relative conversion efficiency of BGHB crystals for output power. It is observed that the conversion efficiency of BGHB is equal to that of KDP crystals. 
Table 1: SHG Conversion of KDP, BGHB

\begin{tabular}{|c|c|c|}
\hline \multicolumn{2}{|c|}{ Output power in Watt } & $\begin{array}{l}\text { Relative conversion } \\
\text { efficiency (SHG in \%) of } \\
\text { BGHB }\end{array}$ \\
\hline KDP & BGHB & 125 \\
\hline 0.6 & 0.75 & 123.5 \\
\hline 0.8 & 0.99 & 116 \\
\hline 1 & 1.16 & 125 \\
\hline 1.2 & 1.49 & 135.4 \\
\hline 1.3 & 1.76 & \\
\hline
\end{tabular}

\section{THERMAL STUDIES}

\subsection{Thermal Gravimetric Analysis}

In order to find the thermal properties of the grown BGHB crystal, Thermo gravimetric analysis (TGA), and Differential thermal analysis were carried out for BGHB crystal. The TGA were carried out between $250 \mathrm{C}$ and $1400 \mathrm{C}$ in nitrogen atmosphere at a heating rate of $100 \mathrm{C} / \mathrm{min}$ using NETZSCH STA $409 \mathrm{C}$. The fig (8) shows the resulting traces of the grown crystal.

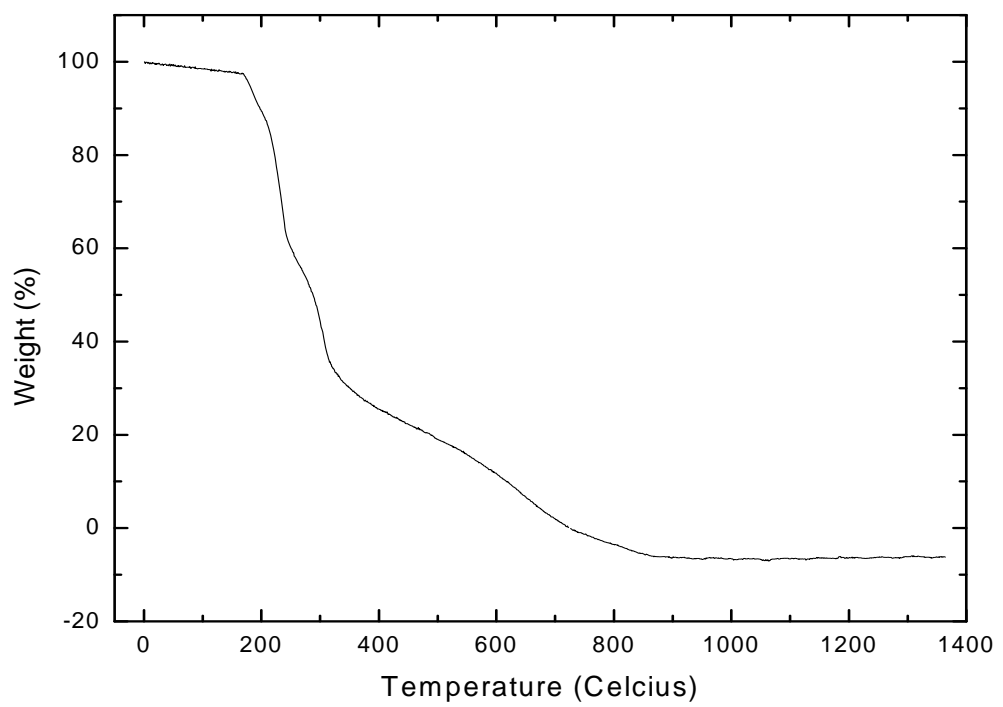

Fig.8: TGA Spectrum of BGHB single crystal 


\subsection{Differential Thermal Analysis}

In order to find the thermal properties of the grown BGHB crystal, Differential thermal analysis (DTA) were carried out for BGHB crystal. The DTA were carried out between $25^{\circ} \mathrm{C}$ and $140^{\circ} \mathrm{C}$ in nitrogen atmosphere at a heating rate of $10^{\circ} \mathrm{C} / \mathrm{min}$ using NETZSCH STA $409 \mathrm{C}$. The Fig (9) shows the resulting traces of the grown crystal.

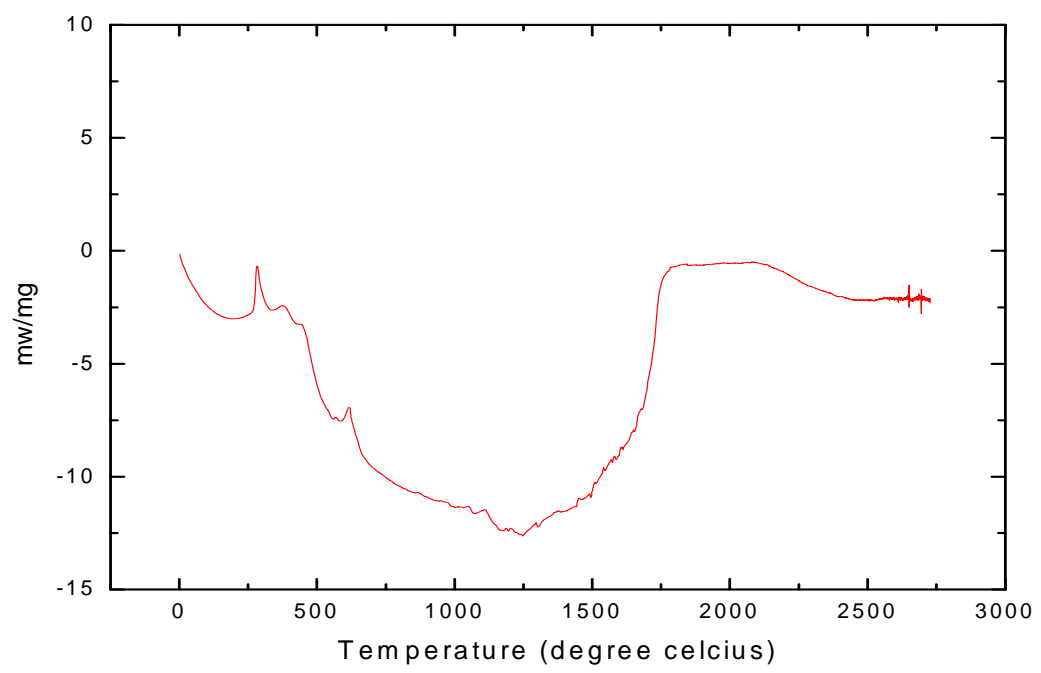

Fig.9: DTA Spectrum of BGHB single crystal

\subsection{Differential scanning calorimetry (DSC)}

In order to find the thermal properties of the grown BGHB crystal, Differential Scanning Calorimetry (DSC) were carried out for BGHB crystal. Show the resulting traces of the grown crystal. The differential scanning Calorimetry (DSC) of BGHB was also carried out between $20^{\circ} \mathrm{C}$ and $200^{\circ} \mathrm{C}$ at a heating rate of $10^{\circ} \mathrm{C} / \mathrm{min}$ in nitrogen atmosphere using NETZSH DSC Z04. It is shown in the Fig (10). 


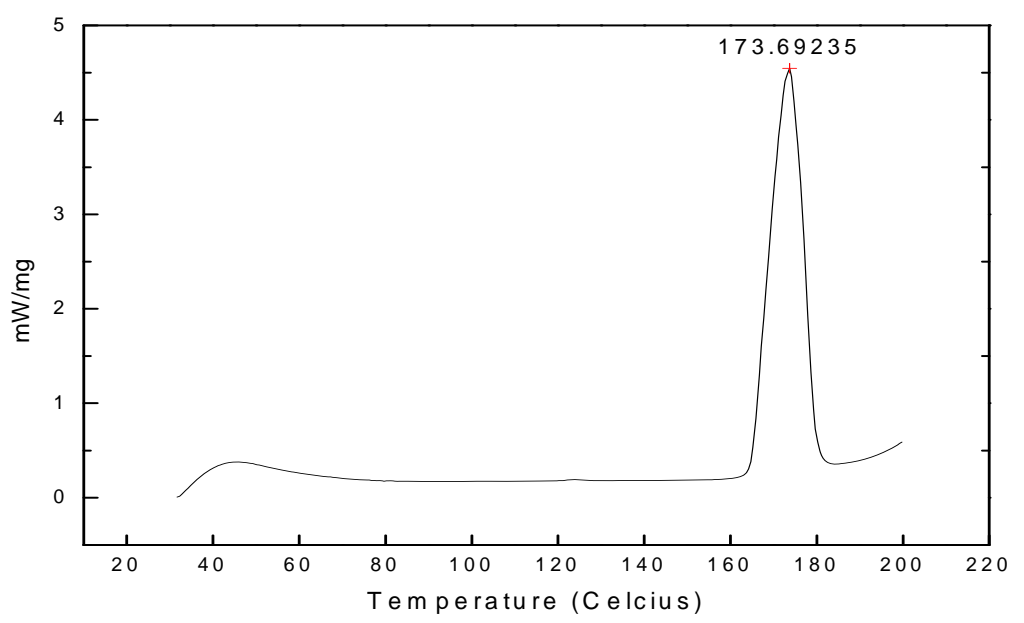

Fig.10.DSC Spectrum of BGHB single crystal

\section{CONCLUSIONS}

Bulk single crystals of Bi-Glycine Hydrogen Bromide (BGHB) have been successfully grown by the slow evaporation technique from the aqueous solution. The solubility curves for the BGHB in de-ionised water, ethanol and methanol at various temperatures have been determined. From the solubility curve, it is concluded that polar solvent like water is opt for growing a bulk size crystal. It is observed from the morphology studies that the growth rate along c direction is faster than other directions. Optical transmission studies confirm that the grown BGHB is transparent in the entire visible region and the FTIR trace reveals the presence of amino groups and functional groups. The NLO studies were carried out and compared with KDP. It is found that the efficiency of BGHB is slightly higher than that of KDP. It is evident from the thermal studies that the crystal is thermally stable up to $168.5^{\circ} \mathrm{C}$ and there is no phase transition and decomposition till $168.5^{\circ} \mathrm{C}$. Anisotropy in the hardness behavior has been observed while measuring at different crystal planes by Vicker hardness test

\section{REFERENCE}

1. D.Eimert, S.Velsko, L.Davis, F.Wang, G.Loiaccono, G.Kennady, IEEE J.Quantum Electron, 25 (1989) 179.

2. D.Xu, M. Jiang, and Z. Tan, Acta Chem. Sin, 41(1983) 570.

3. M. H, Aggarwal, J. Choi, W. S. S Wang, K. Bhat, R. B. Lal, A. D. Shields, B. G. Penn and D. V. Frazier, J. Cryst. Growth,179 (1999) 2004.

4. K.Selvaraju, R. Valluvan, K. Kirubavathi, and S.Kumararamanan, Opt. Comm, 269 (2007) 230 .

5. V. Kannan, R. Bairava Ganesh, and P. Ramasamy, Crystal Growth Des. 6, (2006)1876. 
6. K. V. Rajendran, D. Jayaraman, R.Jayavel, R. Mohan Kumar, and P.Ramasamy, J. Cryst. Growth 224, (2001) 122.

7. K. V.Rajendran, D.Jayaraman, R.Jayavel, P. Ramasamy, J. Cryst. Growth 255, (2003) 361.

8. J.Madhavan, S. Aruna, K. Prabha, J. Packium Julius, p.Joseph Ginson, S.Selvakumar, and S. Sagayaraj, J. Cryst. Growth 293, (2006) 409.

9. K.Selvaraju, R. valluvan, and K. Kumararaman, mater. Let. 60, (2006) 1565.

10. S.K.Kurtz, T.T perry, J. Appl. Phys. 39 (1968) 3798.

11. William Kemp, “Organic Spectroscopy”, Palgrave Macmillan, 1991.

12. R.M. Silverstein, F.X. Webster, Spectrometric Identification of Organic Compounds, $6^{\text {th }}$ Edition, Wiley, New York, 1997 